\title{
Telemedicine Implementation on a Bariatric Outpatient Clinic During COVID-19 Pandemic in Italy: an Unexpected Hill-Start
}

\author{
Matteo Runfola ${ }^{1}$ (D) $\cdot$ Giovanni Fantola ${ }^{1} \cdot$ Stefano Pintus ${ }^{1} \cdot$ Mauro lafrancesco $^{2} \cdot$ Roberto Moroni $^{1}$ \\ Received: 20 May 2020 / Revised: 19 September 2020 / Accepted: 22 September 2020 / Published online: 27 September 2020 \\ (C) Springer Science+Business Media, LLC, part of Springer Nature 2020
}

\begin{abstract}
Background SARS-CoV2 outbreak has challenged NHS of many countries. Generalized restriction of movement, together with unprecedented pressure on Health System, disrupted routine care for non-COVID-19 patients. Telemedicine has been promoted to reduce the risk of infections and to offer medical assistance to the restricted population. This paper is aimed to evaluate the impact of tele-consulting technology in a single bariatric center.

Materials and Methods Our outpatient clinic reorganized the service from on-site to long-distance video consultations. All patients received a satisfaction questionnaire. The main goals were to evaluate patient compliance and to assess patient satisfaction.

Results Of the 33 booked patients who were offered a teleconsultation, 19 (57.6\%) participated in the video-call. No significant differences were found between participants and non-participants in terms of age and gender ratio. Urban area residents were $57.9 \%$ of the participants versus $42.8 \%$ of the non-participants group. Of the participants, $52.6 \%$ completed the survey reporting levels of satisfaction ranging from high to very high.

Conclusion Telemedicine has been advocated as a useful tool to relieve pressure on the overwhelmed Health Systems during the COVID-19 pandemic. However, e-health technologies are not yet widely adopted. Our initial experience, also compared with national data relating to the digital divide, suggests that the absence of basic computer skills and the lack of confidence with video-call systems may be patient-specific barriers for the implementation of telemedicine. In this context, telemedicine implementation can run up against various patient-related barriers, and several challenges remain for e-health to be integrated into outpatient practice.
\end{abstract}

Keywords Telemedicine $\cdot$ COVID-19 $\cdot$ Bariatric surgery $\cdot$ Outpatient $\cdot$ Digital divide

\section{Introduction}

Since January 2020, the outbreak of a new coronavirus originated in China (SARS-CoV-2) imposed a great deal of stress on a growing number of National Health Systems and progressively became a global crisis, to such an extent that on March 11, 2020, the World Health Organization characterized the COVID-19 outbreak as a pandemic [1].

Matteo Runfola

matteorunfola.med@gmail.com

1 Department of Surgery, Obesity Surgery Unit, A.O. Brotzu, Piazzale Alessandro Ricchi, 1, 09134 Cagliari, Italy

2 Cardiovascular Sciences Department, Foundation Polyclinic University A Gemelli IRCCS, Rome, Italy
The Italian Government acted quickly and measures to contain the spread of the infections escalated up to the decision to drastically restrict freedom of movement on the entire Italian population and to close all nonessential businesses and industries on March 21 [2]. On that date, 183,957 total cases and 24,648 deaths were counted throughout the country showing an exponential growth of new diagnoses [3].

Generalized restriction of movement together with the unprecedented pressure on the Health System disrupted routine care for non-COVID-19 patients [4].

Indini et al. [5] described the need for a reorganization of clinical services for non-COVID-19 cancer patients and how this has been addressed in Italy, and Di Saverio et al. [6] showed the same for surgical patients with colonic diseases.

In this context, telemedicine, and in particularly video consultations, has been widely promoted and implemented according to the possibilities of each institution to reduce the 
risk of infection and to offer medical assistance to the restricted population [7].

The provision of bariatric surgery was equally affected by the abovementioned restriction policies, and Bariatric Surgery Centers were challenged with the difficulties to continue to offer their outpatient consultations despite these difficulties.

At our Institution in Cagliari, the largest city of the island of Sardinia, surgical practice has been limited to emergent/urgent cases since March 9, and at the same time, outpatient services have been drastically reduced to minimize the risk of transmission.

Our Bariatric Surgery Center performs approximately 4800 outpatient consultations per year, with more than 400 patients being assessed for the first time as surgical candidates.

Since the beginning of the crisis, our outpatient clinic reorganized its service from on-site to long-distance video consultations for those patients who had previously booked a first appointment to the Bariatric Surgery service for the months of March and April 2020.

Our service was already offering remote consultations by phone, email, or WhatsApp messenger, both in the early postoperative stage (ERAS protocol for bariatric surgery [8]) and in the long-term follow-up. For this reason, the new mobility restrictions had minor impact on those patients who had already undergone surgery, for whom it was not necessary to drastically change the routine.

On the contrary, for patients waiting for a first visit, it was necessary to think about new telemedicine approaches, to avoid leaving them waiting for a non-site visit for an indefinite period of time.

This study will therefore consider our preliminary experience in telemedicine only for patients on their first visit.

\section{Methods}

\section{Patients}

All patients who booked a first clinic appointment were included. The patients were briefly contacted by phone a few days before the scheduled appointment and offered to either keep the date as a video call consultation, or to postpone it to a later time, to attend the visit in person at the clinic. Skype by Microsoft ${ }^{\circledR}$ "Voice over Internet Protocol" (VoIP) software was chosen for video calls because of its simplicity and widespread use.

On the scheduled day of the visit, patients could contact the bariatric surgeon of our Center through a dedicated Skype account. During the video-consultation, a complete medical history was collected and anthropometrical data, measured by patients at home, were recorded.

Patients deemed surgical candidates, in accordance with IFSO guidelines, received preliminary information about the preoperative screening (nutritional and psychological assessment, blood test, instrumental investigations, etc.) during the video call. Additional details, such as surgical information and ERAS protocol counseling, were then provided by email. Patients who did not meet surgical criteria were referred to the nutritionist of our Center, for a specific video call consultation.

\section{Satisfaction Questionnaire}

Following the video-consultation, all patients received a multiple-choice satisfaction questionnaire (SurveyMonkey®) to rate technical and relational aspects and the perceived usefulness of the video call. The answers were articulated in a 5 level scale from "completely negative" to "completely positive" (Table 2).

\section{Endpoints}

The two main goals of the study were to evaluate patient compliance to the video-consultations (drop-out rate before the video call) and to assess patient satisfaction (questionnaire after the video call).

A secondary endpoint was the ability to properly select patients with a correct indication for bariatric surgery.

\section{Statistical Analysis}

Data are presented as count and percentage for categorical variable and median and interquartile range [IQR] for continuous variables. Categorical data were compared using the chisquare test $\left(\chi^{2}\right)$. Continuous variables were compared using the Student's $t$ test.

\section{Results}

From March 9 to April 22, 33 patients who booked their first visit at our Center were offered a video consultation.

Eight patients $(24.2 \%)$ declared from the start that they were not interested in the video consultation. The main reasons to refuse to participate were as follows: "I don't have Skype," "I don't have an email address," and "I don't feel comfortable."

The remaining 25 patients received an email with technical instructions; of these, 19 patients $(57.6 \%)$ participated to the video consultation while 6 did not (overall 14 patients $(42.4 \%)$ dropped-out) (Fig. 1).

No significant differences were found between participants and non-participants in terms of age and gender ratio (Table 1). Urban area residents were $57.9 \%$ in the participants group versus $42.8 \%$ in the non-participants group. On average, consultations lasted $19 \mathrm{~min}$ and $40 \mathrm{~s}$. The satisfaction 
Fig. 1 Participating patients flow diagram

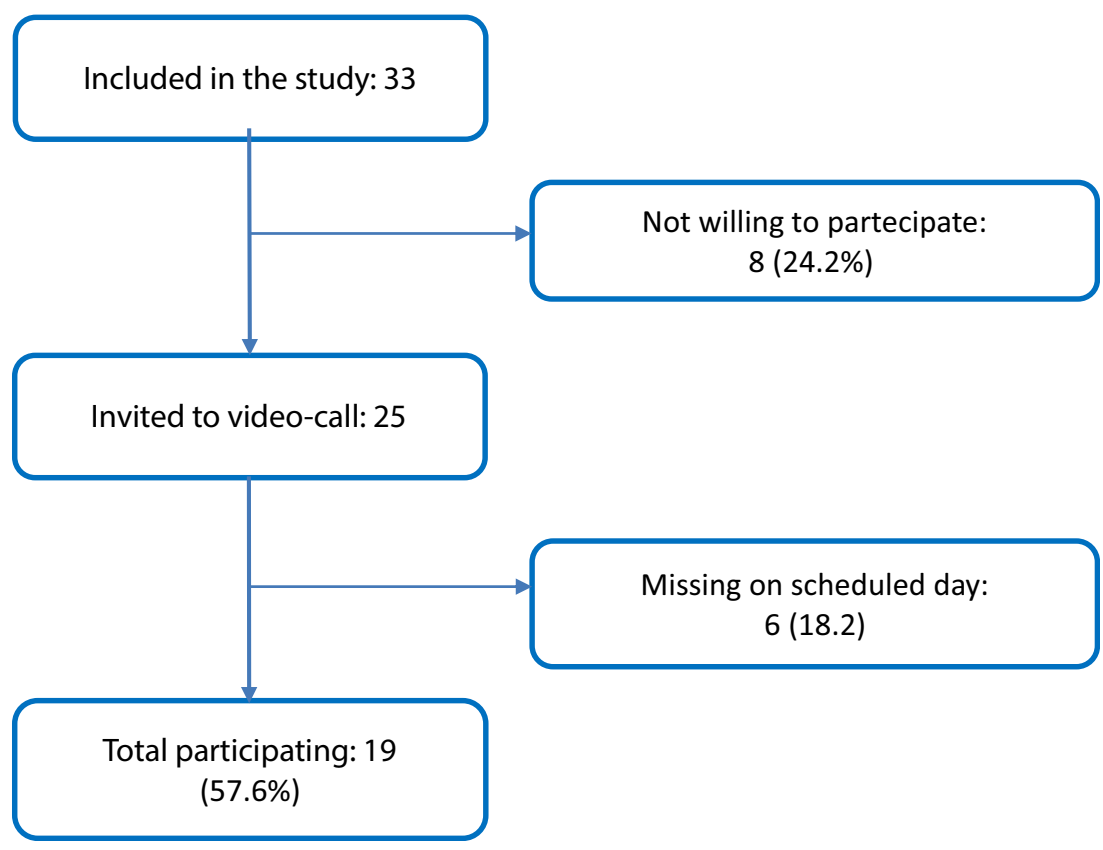

questionnaire was sent by email to all 19 participating patients, but only $10(52.6 \%)$ completed the survey.

The results of the satisfaction survey are summarized in Table 2.

Seventeen patients $(89.5 \%)$ were proposed for bariatric surgery and the subsequent on-site evaluation, performed after the removal of restrictions, confirmed the indication or contraindication for surgery in all cases.

\section{Discussion}

Telemedicine has been strongly advocated as a useful tool to relieve pressure on the overwhelmed Health Systems during the COVID-19 pandemic. The urge to implement e-health systems in this critical phase can turn into an opportunity to reduce structural barriers, especially in disadvantaged areas. The island of Sardinia, with its 1.6 million citizens spread on $24,100 \mathrm{~km}^{2}$, is the second Mediterranean island and the third

Table 1 Demographics

\begin{tabular}{llll}
\hline & Participants & $\begin{array}{l}\text { Non- } \\
\text { participants }\end{array}$ & $P$ \\
\hline$N$ & 19 & 14 & \\
Median age (IQR) & $50(16)$ & $45(18.8)$ & 0.86 \\
M/F ratio & $5 / 14$ & $4 / 11$ & 0.95 \\
Urban area residents (\%) & $10(57.9 \%)$ & $6(42.8 \%)$ & 0.58 \\
\hline
\end{tabular}

Data are presented as count and percentage for categorical variable and median and interquartile range [IQR] for continuous variables
Italian Region for extension, and ranks third last in terms of regional population density.

In this context, we felt that a rapid implementation of a telemedicine service would represent a valid answer to the service disruption that the COVID-19 pandemic has imposed on our Bariatric Surgery Center activity. Nevertheless, preliminary results of this approach showed that patients could find it difficult to adapt to such a system.

Telemedicine offers clear opportunities to abolish barriers of distance and to save time and direct costs. Also, in the field of bariatric surgery, there is a growing interest in the application of e-health systems in the pre- and postoperative period [9]. In the context of the COVID-19 pandemic, facilitating healthcare access by maintaining strict individual protection provisions became a priority to ensure safety for patients and healthcare professionals, and to allow the continuation of specialist medical services.

However, e-health technologies are not yet widely adopted, and many centers had to implement telemedicine services without the appropriate technical knowledge and without the time to train their own staff and properly educate users.

The results of the initial phase of telemedicine in our Bariatric Surgery Center reflect these difficulties. Several patients did not consider video-counseling a useful opportunity and preferred to wait until the end of the mobility restrictions to attend in person to the clinic. Unfortunately, it is not possible at present to predict when this will be possible and it is likely that the use of digital technologies will be a necessity, more than a choice, for an extended period of time.

Moreover, the Italian National Health Service does not recognize telemedicine as an essential level of care, and there lacks a regulatory framework that would allow the integration 
Table 2 Satisfaction questionnaire

\begin{tabular}{|c|c|c|c|c|c|}
\hline \multirow[t]{2}{*}{ Questions } & \multicolumn{2}{|c|}{$\begin{array}{l}\text { Completely } \\
\text { negative }\end{array}$} & \multirow[b]{2}{*}{3} & \multicolumn{2}{|c|}{$\begin{array}{l}\text { Completely } \\
\text { positive }\end{array}$} \\
\hline & 1 & 2 & & 4 & 5 \\
\hline $\begin{array}{l}\text { What was your first reaction towards the possibility of making the visit by } \\
\text { video call? }\end{array}$ & & & & 4 & 6 \\
\hline How did the instructions for making the video call seem to you? & & 1 & & 4 & 5 \\
\hline What was the technical level of the video call (did you hear and see well)? & & & & 7 & 3 \\
\hline How do you evaluate the duration of the conversation? & & & 1 & 4 & 5 \\
\hline Did you feel comfortable asking questions during the call? & & & & 7 & 3 \\
\hline How cordial was the conversation? & & & & 3 & 7 \\
\hline How helpful was the information you received? & & & 1 & 6 & 3 \\
\hline Did you find the information on the surgeries received by email helpful? & & & 1 & 5 & 4 \\
\hline In general, how satisfied are you with the event? & & & & 7 & 3 \\
\hline $\begin{array}{l}\text { How do you evaluate the possibility of making other visits through the } \\
\text { video call in the future? }\end{array}$ & & & & 7 & 3 \\
\hline
\end{tabular}

The answers are articulated in a 5-level scale from "completely negative" to "completely positive." Ten patients returned a $100 \%$ completed questionnaire of e-health platforms into existing electronic medical record system.

Free video-communication solutions (e.g., WhatsApp, Skype, or Facetime) raise questions about privacy and security requirements.

Technological improvements and cost reduction of video communication solutions combined with both high-speed Internet and the mass spread of smartphones make it possible to offer e-health services to a large audience, but high-speed Internet is not equally available to everybody (digital divide).

The annual report on Internet availability drafted by the Italian National Institute of Statistics (ISTAT) [10] showed that in 2019, $76.1 \%$ of households had Internet access and $74.7 \%$ had a broadband connection, but only $67.9 \%$ used the Internet in the 3 months prior to the interview. Most households without home Internet access indicate the lack of computer skills as the main reason $(56.4 \%)$ and $25.5 \%$ do not consider Internet a useful and interesting tool; economic reasons follow. Another discriminating factor is the educational qualification; $94.1 \%$ of families with at least one university graduate component have a broadband connection against $46.1 \%$ of those families whose members have only attended high school. Disparities are also evident between municipalities of different demographic amplitudes: in metropolitan areas, broadband access rates reach $78.1 \%$, while in municipalities of up to 2000 inhabitants, this share drops to $68.0 \%$.

Data from Sardinia (only available until 2018) [11] do not differ significantly from the national ones.

This initial experience is mainly limited by the low number of samples which limits its statistical power; however, our preliminary data suggest that the absence of basic computer skills and the lack of confidence with video call systems may be important patient-specific barriers for the implementation of telemedicine between the most vulnerable social groups of the Italian population.

Although it is not statistically significant, in consideration of the small sample size, we found a greater presence of residents in urban areas in the group of participants, compared with the group of non-participants $(57.9 \%$ vs $42.8 \%$, respectively), comparably with national data.

One of the structural barriers that influenced our initial experience was probably the lack of an electronic system capable of integrating telemedicine software with hospital medical records, which may resolve privacy and security issues, and of a dedicated Internet portal where patients may obtain operative instructions. In fact, our satisfaction survey reported its lowest result at questions on the clarity and usefulness of the instructions received (Table 2).

Messiah et al. [9], in a recent review of eHealth strategies for metabolic and bariatric surgery patients, analyzed 38 published articles between 2011 and 2019 that varied widely in terms of study design and presented several limitations. Despite this, the authors reported overall positive results in terms of feasibility, acceptability, and preliminary effectiveness of eHealth delivery of pre- and postoperative educational materials, knowledge exchange, and social support.

In accordance with our preliminary experience, the authors concluded by stating the importance to take into account key constructs from the socioecological framework including not only intra/interpersonal and developmental factors but also those of the system or setting that provides the eHealth strategy. In this setting, the relationship between care providers and rural areas is crucial because it is right there that patients are more exposed to social, geographical, and economical burdens and are less inclined to access e-health services, as we observed in our preliminary experience. 
Patient education is time- and resource-consuming and yet it is a critical key to implementing telemedicine. A factor one must consider is the initial investment of time and effort to effectively present the option of telemedicine consultation and then train the patient to the use of the necessary software for the first time, as Smith et al. [12] have extensively explained. The authors, suggesting an Implementation Guide for Rapid Integration of an Outpatient Telemedicine Program during the COVID-19 Pandemic, highlight eight necessary elements: among these are an electronic medical record system infrastructure, adequate and flexible audiovisual platforms, patient education, and patient and caregiver participation - the same issues we faced in our preliminary experience.

\section{Conclusion}

The COVID-19 emergency is an occasion to develop new protocols based on digital technologies that can offer lasting solutions to structural problems.

In this context, telemedicine implementation can run up against various patient-related barriers and several challenges remain for e-health to be routinely used and integrated into outpatient practice, such as definition of regulation frameworks, a strategy to implement technical support and a plan guiding healthcare providers to switch to the new paradigm.

\section{Compliance with Ethical Standards}

Conflict of Interest The authors declare that they have no conflict of interest.

Ethical Approval All procedures performed in studies involving human participants were in accordance with the ethical standards of the institutional and/or national research committee and with the Helsinki Declaration and its later amendments, or comparable ethical standards.

Informed Consent Informed consent was obtained from all individual participants to the video calls included in the study.

\section{References}

1. WHO Director-General's opening remarks at the media briefing on COVID-19 - 11 March 2020. Available in: https://www.who.int/ $\mathrm{dg} /$ speeches/detail/who-director-general-s-opening-remarks-at-themedia-briefing-on-covid-19\%2D\%2D-11-march-2020. Accessed 27 Apr 2020

2. Decreto del Presidente del Consiglio Dei Ministri 22 Marzo 2020: Ulteriori Disposizioni Attuative del Decreto-Legge 23 Febbraio 2020, n. 6, Recante Misure Urgenti in Materia di Contenimento e Gestione dell'emergenza Epidemiologica da COVID-19, Applicabili sull'intero Territorio Nazionale. Italian. Available in: http://www. governo.it/sites/new.governo.it/files/dpcm_20200322.pdf. Accessed 27 Apr 2020

3. Epidemiology for Public Health. IstitutoSuperiore di Sanità. COVID-19 Integrated Surveillance Data in Italy. Available in: https://www.epicentro.iss.it/en/coronavirus/sars-cov-2-dashboard. Accessed 27 Apr 2020

4. Remuzzi A, Remuzzi G. COVID-19 and Italy: What Next? Lancet. 2020;395(10231):1225-8.

5. Indini A, Aschele C, Cavanna L, et al. Reorganization of medical oncology departments during the novel coronavirus disease-19 pandemic: a nationwide Italian survey [published online ahead of print, 2020 Apr 6]. Eur J Cancer. 2020;132:17-23.

6. Di Saverio S, Pata F, Gallo G, et al. Coronavirus pandemic and colorectal surgery: practical advice based on the Italian experience. Color Dis. 2020;22(6):625-34.

7. Ohannessian R, Duong TA, Odone A. Global Telemedicine implementation and integration within health systems to fight the COVID-19 pandemic: a call to action. JMIR Public Health Surveill. 2020;6(2):e18810.

8. Thorell A, MacCormick AD, Awad S, et al. Guidelines for perioperative care in bariatric surgery: enhanced recovery after surgery (ERAS) society recommendations. World J Surg. 2016;40(9):2065-83.

9. Messiah SE, Sacher PM, Yudkin J, et al. Application and effectiveness of eHealth strategies for metabolic and bariatric surgery patients: a systematic review. Digit Health [Internet]. 2020;6

10. National Institute of Statistics (ISTAT). Citizens and Information and Communication Technologies 2019 Annual Report. Italian. Available in: https://www.istat.it/it/files//2019/12/Cittadini-e-ICT-2019.pdf. Accessed 27 Apr 2020

11. National Institute of Statistics (ISTAT). Citizens, Enterprises and Information and Communication Technologies 2018 Annual Report. Italian. Available in: https://www.istat.it/it/files/2019/01/ Report-ICT-cittadini-e-imprese_2018_PC.pdf. Accessed 27 Apr 2020

12. Smith WR, Atala AJ, Terlecki RP, et al. Implementation Guide for Rapid Integration of an Outpatient Telemedicine Program during the COVID-19 Pandemic. J Am Coll Surg. 2020;231(2):216-22.

Publisher's Note Springer Nature remains neutral with regard to jurisdictional claims in published maps and institutional affiliations. 